\title{
Development of phenotyping assays for resistance screening of blueberry plants against Neofusicoccum spp.
}

\author{
R.E. Campbell ${ }^{1}$, H. Walters ${ }^{1}$, K.M.S. Tennakoon ${ }^{2}$, E.E. Jones ${ }^{2}$ and M. Walter ${ }^{1}$ \\ ${ }^{1}$ The New Zealand Institute for Plant \& Food Research Ltd, Nelson, Old Mill Road, RD3, \\ Motueka, 7198, New Zealand \\ ${ }^{2}$ Faculty of Agriculture and Life Sciences, Lincoln University, PO Box 85084, Lincoln, \\ New Zealand \\ Corresponding author: Rebecca.campbell@plantandfood.co.nz
}

\begin{abstract}
Botryosphaeria disease is a common problem on a large range of hosts, including blueberry, causing dieback and cankers. Methods for phenotyping assays were developed in this project to facilitate blueberry resistance breeding. Detached shoots were used to test effects of wound size (needle, scalpel), inoculum type (mycelium, conidia), stem hardness (soft, semi-soft) and incubation temperatures on lesion development. Experiments were carried out on various blueberry cultivars with four Neofusicoccum strains, two each of $N$. parvum and $N$. ribis. Lesions of similar lengths developed on both scalpel and needle wounded shoots indicating no wound size effects. Mycelium plugs produced lesions faster than conidial suspension. Similarly, lesions on soft wood progressed faster than those on semisoft cuttings. Incubation temperature had a significant effect on lesion development, with $26^{\circ} \mathrm{C}$ showing faster disease development than 19 or $22^{\circ} \mathrm{C}$. This information will inform assays for blueberry plant breeding for improved disease resistance.
\end{abstract}

Keywords blueberry, Botryosphaeriaceae, disease screening, inoculum, Neofusicoccum, temperature, wounding.

\section{INTRODUCTION}

Botryosphaeria disease causes stem blight dieback, cankers and crown rot, which is estimated to affect $18 \%$ of blueberry plants in main production areas in New Zealand (Sammonds et al. 2009). This disease is increasingly becoming an economically important problem for blueberry production worldwide (Langford 2010; Xu et al. 2015). Crop diseases such as Botryosphaeria reduce yield and decrease the quality and stability of production (IAEA 2010). Botryosphaeria disease has a wide range of susceptible plant hosts (Manawasinghe 2016) with the majority of infections entering through wounds such as pruning cuts or plant injury caused by herbicides, freezing or harvesting (Cline et al. 1993).

While the disease is becoming more economically important, factors such as consumer market, environmental and economic cost and pathogens developing future disease resistance to chemicals, all aim to reduce the reliance on chemicals to control diseases (IAEA 2010). Therefore, an economical and sustainable method of managing disease is the selection of host resistance for improvement of crop production systems (Garg et al. 2008; Spencer \& Lebeda 2010). To enable this, breeders need 
efficient methods to enable successful resistance breeding programmes (IAEA 2010).

Blueberries belong to the genus Vaccinium which is very large and diverse with a number of species contributing genetic material to cultivars grown today. Breeders focus on a number of traits such as fruit yield, fruit flavour, fruit colour, fruit firmness, harvest seasonality and plant growth (Galletta \& Ballington 1996). Various cultivars also have different responses to pathogens with resistant varieties found both in cultivated and wild cultivars of blueberry (Cline et al. 1993). Additionally, pathogen species and isolates vary in pathogenicity, with Neofusicoccum ribis and $N$. parvum found to be the most pathogenic Botryosphaeria species in New Zealand (Tennakoon et al. 2017).

Using pathogen isolates as selection agents to screen host plants for resistance is now well established (Lebeda \& Švábová 2010) and there are various controlled-environment screening methods used to evaluate resistance (Garg et al. 2008). Cline et al. (1993) used potted blueberry plants inoculated with Botryosphaeria to screen for host resistance, which required at least one month to get results and large areas for plant incubation. In vitro tests are able to provide more controlled climatic conditions, precise methods and enable testing of a large number of individuals in a small space (Lebeda \& Švábová 2010). Screening of populations for disease resistance requires wound inoculation of attached or detached stems where the lesion length from the point of inoculation is used to determine the degree of resistance (Smith 1997). Individual plant performance can vary depending on the test method used for comparing the level of resistance (Garg et al. 2008); therefore it is important that mass screening methods should mimic the infection process under natural conditions (IAEA 2010). It has previously been shown that stem age, isolate virulence, inoculation environment and evaluation timing are critical factors in determining resistance (Cline et al. 1993). However, for inoculation of Botryosphaeria in blueberries, only mycelium plugs have been widely used and little is known about the alternative, more natural infection method using spore inoculum. Therefore, to test host susceptibility and lesion development, factors such as wound type, inoculum type, plant material and temperature need to be taken into account to screen different blueberry cultivars based on their susceptibility to Botryosphaeria disease.

The aim of this study was to undertake a set of experiments to determine suitable methods for effective phenotyping assays for blueberry infections with Neofusicoccum. The susceptibility of a number of blueberry cultivars were assessed inoculating with various Neofusicoccum isolates while investigating the role of incubation temperature, wound size, inoculum method and stem hardness.

\section{MATERIALS AND METHODS}

Fungal isolates, spore production and cultivars Neofusicoccum isolates used in this study were originally isolated from blueberry in a New Zealand nationwide survey (Tennakoon 2017) and obtained from the Lincoln University culture collection. Two species, each with two isolates, were used - N. ribis (LUPP1348, LUPP1365) and N. parvum (LUPP1288, LUPP1363). Fungal isolates were grown and maintained on Potato dextrose agar (PDA) (Difco ${ }^{\mathrm{TM}}$ ) at $22^{\circ} \mathrm{C}$. These cultures were sub-sampled no more than four times, and plugs for inoculation were taken from the growing margin of a 5-6 day old culture. Each experiment used plugs from only one subsample of the same age for all treatments. Fresh spore suspensions were generated by washing spores off infected twigs that had been dried and rehydrated in sterile water following the methods of Tennakoon et al. (2017). The concentrations were adjusted using a haemocytometer For each experiment, up to 10 blueberry cultivars were selected from a total of 12 from Vaccinium corymbosum (highbush; 'Blue bayou', 'Sunset blue') and V. ashei (rabbiteye; 'Dolce blue', 'Tiff Blue', 'Centra blue', 'Centurion', 'Maru', 'Ocean blue', 'Powder blue', 'Rahi', 'Velluto blue', 'Sky blue'). These cultivars were made available from The New Zealand Institute for Plant \& Food Research Limited (PFR), Motueka. Plants were grown outdoors as part of the PFR germplasm 
collection under bird netting, in a sawdustsoil mix in raised beds. Plants were given solid fertiliser (no fertigation) and watered using dripper lines as per commercial production. Herbicides were used to control weeds around plants and no fungicides or insecticides were applied. These plants did not have any previous Botryosphaeria exposure.

\section{Shoot incubation}

Soft green and semi-hardened (semi-soft) detached shoots of various blueberry cultivars from field-grown blueberry plants were harvested using sterilised secateurs.

All leaves were removed from stems except for 3-4 at the top of soft, and 2-3 on semi-soft shoots. Shoots were cut to $15 \mathrm{~cm}$ in length and gently washed in cold water to remove any dirt from the field. The shoots were surface sterilised by swabbing with $70 \%$ ethanol. Shoots were placed into sterilised glass universals containing sterilised distilled water and the top of the universal was wrapped with plastic paraffin film to support the shoot and prevent evaporation and spillage.

Wounds were created using a sterile scalpel to create one wound (roughly $5 \mathrm{~mm}$ in length) halfway up the stem (below the leaves) or by using a sterile needle to make three small wounds $2 \mathrm{~cm}$ apart on each stem, also halfway up the stem below the leaves.

For each experiment, shoots were inoculated with one or more Neofusicoccum isolate using either mycelium plugs or spore suspension. Mycelium plugs were cut from the growing edge of the PDA plates using a 3-mm cork borer and transplanted onto the wound using a sterile scalpel. Control shoots were inoculated by the same method with sterile PDA plugs. Wounds were lightly misted with water beforehand to help the plug stay in place. For spore inoculation, a $10 \mu \mathrm{L}$ droplet of spore suspension was pipetted onto the fresh wound. Control shoots were inoculated with $10 \mu \mathrm{L}$ sterile distilled water pipetted onto the fresh wound.
After inoculation, shoots were immediately placed into airtight, see-through plastic containers (30 litre Sistema boxes) which had been misted with water to create a humid environment (Smith 1997). These containers were incubated at $22^{\circ} \mathrm{C}$ for Experiment $1-5$, and 19,22 and $26^{\circ} \mathrm{C}$ for Experiment 6 in three different laboratories. The containers were lightly sprayed with water for the first 3 days after inoculation to maintain humidity (Smith 1997; Tennakoon et al. 2017) before the lids were slightly removed to slightly reduce the humidity and shoots to dry out. Signs of infection were usually observed 2-7 days post-inoculation. Symptoms were observed as wilting and dieback of shoots and dark brown/ black lesions which spread along the stems in some cases onto the remaining leaves. Lesion lengths (LL) were measured (assessments) using a digital calliper for 1-3 weeks depending on the experiment. Lesions were confirmed to be caused by Botryosphaeria by re-isolating spores from the dying shoots by drying and rehydrating the shoots in sterile water and identifying the spores under a microscope.

\section{Experimental design}

All experiments were replicated in a randomised complete block design. Each factor was randomised over a number of plastic boxes in a balanced design, with the exception of the different isolates, which were contained in different boxes to prevent cross contamination. The variables, treatments and replication for each experiment are shown in Table 1. The number of replicates was split equally over the treatment factors for the specific experiments, resulting in equal numbers of replicate shoots for each combination of factors. All experiments had either blank PDA plugs or sterile water controls with the same number of shoots as the treatment factors. 
Table 1 Variables for the six experiments to investigate methods for screening Blueberry (Vaccinium corymbosum (highbush, indicated by ${ }^{*}$ ) and V. ashei (rabbiteye) for susceptibility to Botryosphaeria Neofusicoccum strains (N. ribis: LUPP1348, LUPP1365 and N. parvum: LUPP1288, LUPP1363). The number of replicates was split equally over the treatment factors to create a balanced design. For example, Experiment 6 in the far right column had 60 replicate shoots, split over two isolates and three temperatures, resulting in 10 shoots for each isolate at each temperature. $¥$ Note that isolates were in separate boxes to avoid cross contamination.

\begin{tabular}{|c|c|c|c|c|c|c|}
\hline \multirow[b]{2}{*}{ Variables } & \multicolumn{6}{|c|}{ Experiment number } \\
\hline & 1 & 2 & 3 & 4 & 5 & 6 \\
\hline $\begin{array}{l}\text { Number of } \\
\text { replicates } \\
\text { (shoots) }\end{array}$ & 100 & 58 & 40 & 160 & 200 & 60 \\
\hline \multirow[t]{12}{*}{ Cultivars } & 'Dolce blue' & 'Dolce blue' & 'Dolce blue' & 'Dolce blue' & 'Dolce blue' & - \\
\hline & 'Tiff blue' & - & - & - & 'Tiff blue' & - \\
\hline & - & - & - & 'Blue bayou’* & - & - \\
\hline & - & - & - & 'Centra blue' & - & - \\
\hline & - & - & - & 'Centurion' & 'Centurion' & - \\
\hline & - & - & - & 'Maru' & 'Maru' & - \\
\hline & - & - & - & 'Ocean blue' & 'Ocean blue' & - \\
\hline & - & - & - & 'Powder blue' & 'Powder blue' & - \\
\hline & - & - & - & 'Rahi' & 'Rahi’' & 'Rahi’ \\
\hline & - & - & - & - & 'Sky blue' & - \\
\hline & - & - & - & - & $\begin{array}{l}\text { 'Sunset } \\
\text { blue» }\end{array}$ & - \\
\hline & - & - & - & - & 'Velluto blue' & - \\
\hline \multirow[t]{4}{*}{ Isolates } & LUPP1288 & LUPP1288 & LUPP1288 & LUPP1288 & LUPP1288 & LUPP1288 \\
\hline & - & - & LUPP1363 & - & LUPP1363 & - \\
\hline & LUPP1365 & LUPP1365 & LUPP1365 & LUPP1365 & LUPP1365 & LUPP1365 \\
\hline & - & - & LUPP1348 & - & LUPP1348 & - \\
\hline \multirow{2}{*}{$\begin{array}{l}\text { Inoculation } \\
\text { type }\end{array}$} & Mycelium & - & Mycelium & - & Mycelium & - \\
\hline & Spores & Spores & - & Spores & - & Spores \\
\hline \multirow[t]{2}{*}{ Tissue type } & Soft & Soft & Soft & Soft & - & Soft \\
\hline & Semi-soft & Semi-soft & Semi-soft & Semi-soft & Semi-soft & - \\
\hline \multirow{3}{*}{$\begin{array}{l}\text { Temperature } \\
\left({ }^{\circ} \mathrm{C}\right)\end{array}$} & 22 & 22 & 22 & 22 & 22 & 19 \\
\hline & & & & & & 22 \\
\hline & & & & & & 26 \\
\hline $\begin{array}{l}\text { Assessments } \\
\text { (weekly) }\end{array}$ & 2 & 3 & 3 & 2 & 1 & 2 \\
\hline \multirow[t]{2}{*}{ Wound } & Scalpel & Scalpel & Scalpel & Scalpel & Scalpel & - \\
\hline & - & Needle & $\ldots$ & - & - & Needle \\
\hline $\begin{array}{l}\text { Transforma- } \\
\text { tion of lesion } \\
\text { length data }\end{array}$ & $\log$ & $\log$ & None & None & None & $\log$ \\
\hline
\end{tabular}




\section{Statistical analyses}

General analysis of variances (ANOVA) were carried out in R ( $\mathrm{R}$ Core Team 2016) separately for each of the experiments. The response variable of some experiments needed transformation to meet the statistical assumptions (Table 1). All $\mathrm{P}$ values, unless otherwise stated, are for the full models with all factors relevant to the particular experiment as listed in Table 1. Comparisons between individual treatments to detect statistical difference between group means were made using Tukey's Honest Significant Difference (HSD) test (Quinn \& Keogh 2002). Significance values corresponding to the Tukey's HSD tests are displayed as adjusted $\mathrm{P}$ values $\left(\mathrm{P}_{\mathrm{adj}}\right)$. The stem location of the three needle wounds did not have a significant effect on lesion length ( $\mathrm{LL}, \mathrm{P}=0.118$, Experiment $2 ; \mathrm{P}=0.157$, Experiment 6); therefore, only the middle wound was analysed as a comparison to scalpel wounds.

\section{RESULTS}

\section{Inoculum, tissue type and assessment timing}

Lesion length was significantly longer $(\mathrm{P}<0.001)$ when a mycelium plug was used as inoculum as opposed to a spore solution, after the same length of time for infection to develop (Experiment 1, Table 2). There was an obvious effect of tissue type, with soft wood showing significantly higher susceptibility than semi-soft wood (Table 2) except in Experiment 2, where lesions developed more slowly. The combination of soft tissue and mycelium plugs could not be differentiated between cultivars as the lesions progressed aggressively. Since the more susceptible cultivars were completely colonised after 3 weeks, comprehensive assessments were best carried out after 1 week for mycelium inoculation and after 2 weeks for spore inoculation. Experiment 2 progressed slower; therefore a complete assessment was possible after 3 weeks. The LL at disease onset from mycelium plug inoculations differed more between tissue types than when inoculated with spores (Experiment 1, Tissue ${ }^{\star}$ Inoculum, $\mathrm{P}<0.001$ at assessment 1); however this interaction between tissue type and inoculum type was no longer significant by assessment $2(\mathrm{P}=0.79)$.

Table 2 Factors influencing lesion length (LL) for all six experiments using up to 10 different blueberry cultivars and up to four Neofusicoccum isolates. See Table 1 for details of experimental design. P values are all from the full model including interaction terms, for each experiment. $\$$ Note that isolates were in separate boxes to avoid cross contamination, therefore not completely randomised and results should be treated with caution.

\begin{tabular}{llclc}
\hline Factor & Description & Experiment & Mean LL $(\mathrm{mm})$ & P-value \\
\hline Inoculum & Mycelium, Spores & 1 & $41.9,10.6$ & $<0.001$ \\
Tissue & Soft, Semi-soft & 1 & $36.9,21.4$ & $<0.001$ \\
& & 2 & $3.0,3.0$ & 0.33 \\
& & 3 & $73.8,27.5$ & $<0.001$ \\
Temperature & $19,22,26^{\circ} \mathrm{C}$ & 4 & $38.2,6.1$ & $<0.001$ \\
Cultivar & 2-10 cultivars & 6 & $15.4,12.8,22.5$ & 0.007 \\
& & 1 & See Figure 1 & 0.04 \\
Isolate $\neq$ & 4 & See Figure 1 & $<0.001$ \\
& 2-4 isolates & 5 & See Figure 1 & $<0.001$ \\
& LUPP1365, & 1 & $30.65,27.6$ & $<0.001 \ddagger$ \\
& LUPP1288, & 2 & $2.2,3.7$ & $0.05 \ddagger$ \\
& LUPP1363, & & & $<0.001 \ddagger$ \\
& LUPP1348 & & & $0.008 \ddagger$ \\
& & 3 & $53.9,54.7,43.2,50.7$ & $0.02 \ddagger$ \\
Wound & & 4 & $26.5,17.6$ & $<0.001 \ddagger$ \\
\hline
\end{tabular}




\section{Wound size}

Wound size (needle versus scalpel) made no significant difference to disease development (Table 2).

\section{Isolates}

When comparing isolates, it needs to be acknowledged that each isolate was contained in a separate box to avoid cross contamination so isolate could, therefore, be confounded with the box they were in. However, there were no trends in the residuals and no effects on the controls that indicate any significant box effect. This study found no significant interactions between cultivars and isolates (Experiment 1, $\mathrm{P}=0.38$; Experiment 4, $\mathrm{P}=0.12$; Experiment $5, \mathrm{P}=0.77$ ). Although the isolates LUPP1288 and LUPP1365 performed significantly different to each other in Experiment 4 and 6, these differences were not observed in Experiment 2, 3 or 5. A significant isolate effect (Table 2) was observed in both Experiments 3 and 5 among the four isolates used, however, this isolate effect was small and not consistent between experiments. All isolates except LUPP1363 produced similar LL in Experiment 3, whereas LUPP1348 produced significantly longer lesions than the other isolates in Experiment 5. In contrast, LUPP1365 infected shoots showed more symptoms than LUPP1288 in both Experiments 4 and 6 (Table 2).

\section{Incubation temperature}

Both isolates tested at various temperatures showed larger lesion development at $26^{\circ} \mathrm{C}$ (27 and $17 \mathrm{~mm}$ for LUPP1365 and LUPP1288 respectively), than 19 and $22^{\circ} \mathrm{C}$ (Table 2). Average $\mathrm{LL}$ at $19^{\circ} \mathrm{C}$ was 16 and $10 \mathrm{~mm}$, and at $22^{\circ} \mathrm{C} 18$ and $7 \mathrm{~mm}$ for LUPP1365 and LUPP1288 respectively.

\section{Susceptible cultivars}

As mentioned in the section 'Isolates', there was no significant interaction between cultivar and isolate (Experiment 4 and 5 respectively, $\mathrm{P}=0.12, \mathrm{P}=0.77$ ). 'Sunset blue' was the least susceptible cultivar tested, followed by 'Blue bayou' and 'Centurion'. Both 'Velluto blue' and 'Sky blue' showed the highest susceptibility while 'Rahi', 'Maru', 'Dolce blue', 'Powder blue' and 'Tiff blue' showed moderate susceptibility (Figure 1). Of the six cultivars repeated in both experiment 4 and 5, five had repeatable relative susceptibility determined by LL, whereas 'Ocean blue' had greater relative susceptibility to mycelium in Experiment 5 than to spores in Experiment 4 (Figure 1).

'Centra blue' had a slower initial onset of disease (e.g. 'Centra blue' cf. 'Maru' assessment 1, $\mathrm{P}_{\mathrm{adj}}<0.0001$ and assessment 2, $\mathrm{P}_{\mathrm{adj}}=0.07$; Experiment 4, Figure 1a, 1b). The lesions on semisoft tissue did not progress as much as those on the soft wood $(\mathrm{P}<0.001)$ when inoculated with spores, resulting in a large number of lesions still small by assessment 2 , however these are lumped in Figure 1a to show the cultivar differences (Experiment 4).

\section{DISCUSSION}

This study explored methods to investigate the blueberry-botryosphaeria pathosystem with the intention to guide the development of screening methods for disease resistant cultivars. The methods used were fast, easy and effective which would enable testing a number of cultivars efficiently.

The methods with forced wounds and virulent isolates could be considered a harsh test of resistance compared to natural infection (Cline et al. 1993). However, conditions that are too harsh for the plant are also not useful for screening resistance (Lebeda \& Švábová 2010). For example, the combination of mycelium plugs and soft shoots in our experiments caused lesion development to progress too quickly, killing the majority of shoots before they could be assessed to determine differences in lesion development between cultivars. Smith (1997) used semisoft tissue with mycelium plugs and was able to efficiently detect differences in susceptibility after $20-30$ days by measuring LL. Whereas, in our experiments mycelium plugs produced large enough lesion growth and separation between cultivars by one week of incubation $\left(22-26^{\circ} \mathrm{C}\right)$ and spores after 2 weeks on soft wood, thus indicating our methods produced faster results for screening, both on soft and semi-soft tissue. 

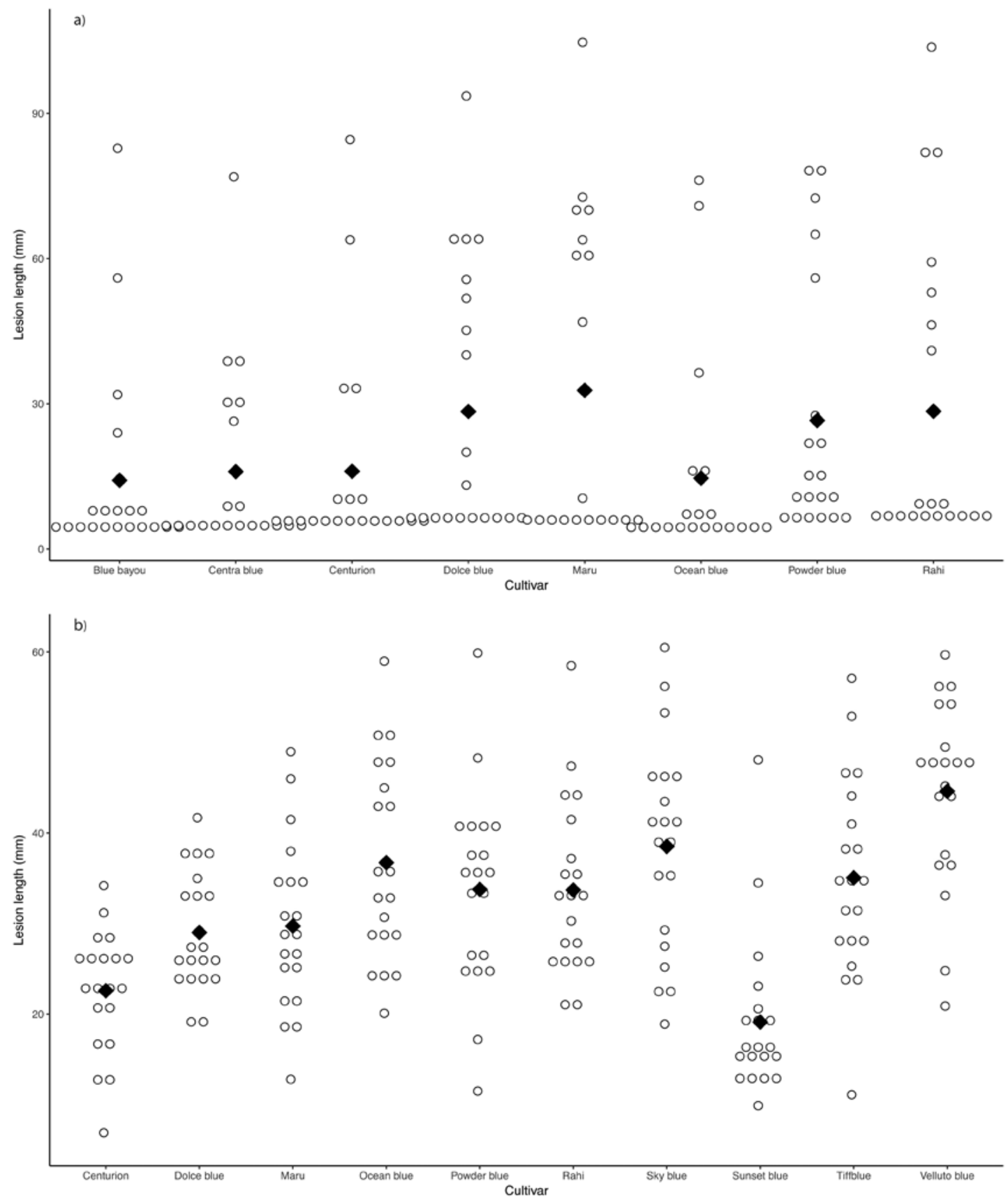

Figure 1 Lesion length in various blueberry cultivars inoculated with both Neofusicoccum ribis and $N$. parvum using (a) spores (Experiment 4, assessment 2, semi-soft and soft shoots) and (b) mycelium (Experiment 5, assessment 1, semi-soft shoots) incubated at $22^{\circ} \mathrm{C}$. The hollow circle dots are the raw data points, with the means shown as black diamonds. 
One important feature of a screening programme is that the pressures put on the cultivars are realistic (IAEA 2010). Because Botryosphaeria fungal spores are primarily rain dispersed (Amponsah et al. 2009), using spore suspensions for inoculation would be more comparable to infection processes in the field. In a spore suspension, it is also possible to mix different isolates of Neofusicoccum, which could be more representative of field conditions where multiple isolates are present at one time (Tennakoon 2017). While previous studies have focussed on using mycelium plugs (Cline et al. 1993; Smith 1997), our study showed success in differentiating between cultivars using spore inoculum in combination with soft wood cuttings and warm temperatures $\left(22-26^{\circ} \mathrm{C}\right)$, to get fast ( 2 weeks) tests of resistance/susceptibility.

Our results show soft green shoots developed more severe symptoms in a shorter time period than semi-soft shoots, which is consistent with the findings of Tennakoon et al. (2017). Yang (2017) has described an easy method of producing a supply of Neofusicoccum spores for spore suspension, which makes this method realistic to produce enough inoculum efficiently. However, if only semi-soft wood is available, mycelium plugs provide a faster test on the older tissue type than spore suspension. Furthermore, if semi-soft shoots are being used as propagation material, this may be a realistic growth stage to use as screening material and easily available. These methods with semi-soft cuttings and mycelium plugs did discriminate well between cultivars, quickly and at all temperatures tested.

While Smith (1997) found significant interactions between cultivars and isolates, our experiments were unable to show any such interactions, thus mixing isolates in spore solutions may be realistic. Previous work (Cline et al. 1993), indicated high pathogen variability but constant relative levels of cultivar susceptibility. In our study, we had relatively stable pathogen virulence in addition to mostly repeatable cultivar relative susceptibilities. Of the six cultivars that were used in both Experiments 4 and 5, five had repeatable results in relative lesion growth, while 'Ocean blue' had less lesion expansion in Experiment 4 using spore inoculation than in Experiment 5 using mycelium plugs. There may be an indication that the 'highbush' (Vaccinium corymbosum) varieties could be less susceptible to Botryosphaeria than the 'rabbiteye' ( $V$. ashei). However, only two highbush varieties were included, therefore further testing would be necessary.

In conclusion, these experiments refined fast, easy and efficient methods to be used in future studies investigating blueberry cultivars and their susceptibility to Neofusicoccum species. Notably, the effectiveness of spore inoculations in conjunction with soft wood cuttings would provide a more realistic approach to inducing infection than previously used mycelium plugs (on semi-soft tissue). The spores can be delivered in liquid suspension in a more realistic manner. Where shoots are in short supply, multiple needle wounds can be created per shoot to produce just as effective inoculation sites as larger wounds. In addition, we found that the lesions (tested on 'Rahi') developed faster at warmer temperatures up to $26^{\circ} \mathrm{C}$. We would, therefore, recommend the use of spore inoculation on soft-wood cuttings using small needle wounds which are then incubated around $26^{\circ} \mathrm{C}$.

\section{ACKNOWLEDGEMENTS}

This work was funded by the New Zealand Institute for Plant \& Food Research Ltd as part of a work-placement of Holly Walters. Thanks to Janice Turner for access to plant material, and Jo Stephens and Bhanupratap Vanga for refereeing.

\section{REFERENCES}

Amponsah NT, Jones EE, Ridgway HJ, Jaspers MV 2009. Rainwater dispersal of Botryosphaeriaceae conidia from infected grapevines. New Zealand Plant Protection 62: $228-233$.

Cline WO, Milholland RD, Rooks SD, Ballington JR 1993. Techniques in breeding for resistance to blueberry stem blight caused by Botryosphaeria dothidea. Acta Horticulturae 346: 107-110. 
Creswell TC, Milholland RD 1988. Spore release and infection periods of Botryosphaeria dothidea on blueberry in North Carolina. Plant Disease 72: 342-346.

Galletta GJ, Ballington JR 1996. Blueberries, Cranberries and Lingonberries. In: Janick J, Moore J eds. Fruit Breeding. Vol. 2 Vine and Small Fruits. J. Wiley \& Sons, New York.

Garg H, Sivasithamparam K, Banga SS, Barbetti MJ 2008. Cotyledon assay as a rapid and reliable method of screening for resistance against Sclerotinia sclerotiorum in Brassica napus genotypes. Australasian Plant Pathology 37: 106. doi:10.1071/AP08002.

International Atomic Energy Agency 2010. Mass screening techniques for selecting crops resistant to disease. International Atomic Energy Agency, Vienna. 327 p.

Langford G 2010. Identification of insects, diseases and disorders in New Zealand blueberries: A manual to aid identification and Control. The New Zealand Institute for Plant and Food Research.

Lebeda A, Švábová L 2010. In vitro screening methods for assessing plant disease resistance. In: Mass screening techniques for selecting crops resistant to disease. International Atomic Energy Agency, Vienna. 327 p.

Manawasinghe IS, Phillips AJL, Hyde KD, Chethana KWT, Zhang W, Zhao WS, Yan JY, Li XH, 2016. Assessing the aggressiveness of plant pathogenic Botryosphaeriaceae. Mycosphere 7 (7): 883-892.

Quinn GP, Keogh MJ 2003. Experimental design and data analysis for biologists. Cambridge University Press, Cambridge. 537 p.

$\mathrm{R}$ Core Team 2016. R: A language and environment for statistical computing. $\mathrm{R}$ Foundation for Statistical Computing, Vienna, Austria. v3.3.2, URL https://www.Rproject.org/.
Sammonds J, Billones R, Rocchetti M, Ridgway HJ, Walter M, Jaspers MV 2009. Survey of blueberry farms for Botryosphaeria dieback and crown rot pathogens. New Zealand Plant Protection 62: 238-432.

Smith BJ 1997. Detached stem assay to evaluate the severity of stem blight of Rabbiteye blueberry (Vaccinium ashei). Acta Horticulturae 446: 457-464.

Spencer M, Lebeda A 2010. Improvement of crop production and disease resistance through mutation induction: The IAEA mandate. In: Mass screening techniques for selecting crops resistant to disease. International Atomic Energy Agency, Vienna. 327 p.

Tennakoon KMS 2017. Botryosphaeria disease in New Zealand blueberry gardens: identification of pathogens, inoculum sources and factors affecting disease development. Lincoln University, Lincoln, New Zealand. $\mathrm{PhD}$ thesis.

Tennakoon KMS, Ridgway HJ, Jaspers MV, Jones EE 2017. Production of Neofusicoccum species conidia and their pathogenicity on wounded and non-wounded blueberry shoots. New Zealand Plant Protection 70: 209-214.

Xu CN, Zhang HJ, Zhou ZS, Hu TL, Wang ST, Wang YN, Cao KQ 2015. Identification and distribution of Botryosphaeriaceae species associated with blueberry stem blight in China. European Journal of Plant Pathology 143(4): 737-752.

Yang YL, Turner J, Stephens J, Campbell RE, Walter M. 2017. Comparison of in vitro and in planta sporogenesis in Neofusicoccum species from blueberry. New Zealand Plant Protection 70: 203-208. 